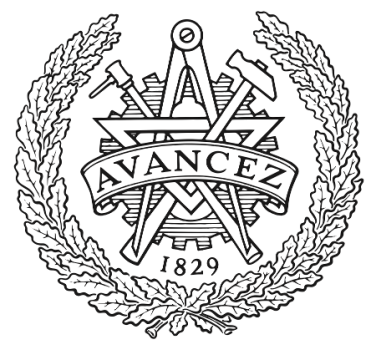

CHALMERS

UNIVERSITY OF TECHNOLOGY

\title{
Performance Analysis for Autonomous Vehicle 5g-Assisted Positioning in GNSS-Challenged Environments
}

Downloaded from: https://research.chalmers.se, 2023-04-26 00:56 UTC

Citation for the original published paper (version of record):

Abu-Shaban, Z., Seco-Granados, G., Benson, C. et al (2020). Performance Analysis for Autonomous Vehicle 5g-Assisted Positioning in GNSS-Challenged Environments. 2020 IEEE/ION Position, Location and Navigation Symposium, PLANS 2020: 996-1003. http://dx.doi.org/10.1109/PLANS46316.2020.9109885

N.B. When citing this work, cite the original published paper. 


\section{Performance Analysis for Autonomous Vehicle 5G-Assisted Positioning in GNSS-Challenged Environments}

\author{
Zohair Abu-Shaban \\ University of New South Wales \\ Canberra, Australia \\ zohair.abushaban@unsw.edu.au \\ Craig R. Benson \\ University of New South Wales \\ Canberra, Australia \\ c.benson@unsw.edu.au
}

\author{
Gonzalo Seco-Granados \\ Universitat Autònoma de Barcelona \\ Barcelona, Spain \\ gonzalo.seco@uab.cat \\ Henk Wymeersch \\ Chalmers University of Technology \\ Gothenburg, Sweden \\ henkw@chalmers.se
}

\begin{abstract}
Standalone Global Navigation Satellite Systems (GNSS) are known to provide a positioning accuracy of a few meters in open sky conditions. This accuracy can drop significantly when the line-of-sight (LOS) paths to some GNSS satellites are obstructed, e.g., in urban canyons or underground tunnels. To overcome this issue, the general approach is usually to augment GNSS systems with other dedicated subsystems to help cover the gaps arising from obscured LOS. Positioning in 5G has attracted some attention lately, mainly due to the possibility to provide cm-level accuracy using $5 \mathrm{G}$ signals and infrastructure, effectively imposing no additional cost. In this paper, we study the hybridization of GNSS and 5G positioning in terms of achievable position and velocity error bounds. We focus on scenarios where satellite visibility is constrained by the environment geometry, and where the GNSS and 5 G positioning systems fail to perform individually or provide prohibitively large error.
\end{abstract}

\section{INTRODUCTION}

Standalone code-driven Global Navigation Satellite Systems (GNSS) positioning methods provide an accuracy of a few meters. When augmented with complementary systems that provide carrier-phase reference signals such as Real-Time Kinematic (RTK) and Precise Point Positioning (PPP), position accuracy goes down to cm-level [1]. However, such augmentation systems suffer from two main shortcomings [2] i) The convergence time for a position fix tends to be long, ii) they are prone to intermittent reception where carrierphase information may be lost, warranting a new position fix calculation. Therefore, we alternatively propose the soon-tobe ubiquitous $5 \mathrm{G}$ millimeter-wave (mmWave) communication technology [3]-[6] to assist GNSS receivers to cover the

Copyright (c) 2020 IEEE. Personal use of this material is permitted. However, permission to use this material for any other purposes must be obtained from the IEEE by sending a request to pubs-permissions@iee.org. G. Seco-Granados was supported in part by the Spanish Ministry of Science, Innovations and Universities through projects TEC2017-89925-R and TEC2017-90808-REDT. Henk Wymeersch was supported by the Swedish Research Council under grant 2018-03701. gaps arising from intermittent GNSS reception and/or provide backup system that could take over the positioning task in GNSS-challenged scenarios, such as urban canyons, indoors, underground tunnels or malicious jamming attacks.

Motivated by the ever-increasing applications requiring location-awareness, many recent studies investigated $5 \mathrm{G}$ positioning with mmWave transmission [7]-[9]. All these studies showed that $5 \mathrm{G}$ standalone systems can provide $\mathrm{cm}$-level positioning accuracy. $5 \mathrm{G}$ employs antenna arrays at the base stations and user equipment, and hence, high-accuracy positioning can be performed with a single base station through the estimation of the range or pseudorange (PR) and the directions of arrival and departure (DOA, DOD) [8]-[10]. MmWave channels are highly sparse and 5G positioning would be viable in environments with many reflections, in which GNSS would fail or have a very poor performance. With all the advantages of $5 \mathrm{G}$, it is highly sensitive to synchronization accuracy and may not be fully available in non-urban areas. Moreover, GNSS may still be the favorable method of positioning in some scenarios, especially in open areas and highways. Therefore, positioning based on synergies of GNSS and 5G helps solve the shortcomings of the individual systems, while retaining and improving the strengths of each. As a use case, we propose this hybridization for systems of autonomous vehicles (AVs), which will be equipped with $5 \mathrm{G}$ transceivers, in any case, making integrating 5G with GNSS a natural solution.

In this paper, we focus on scenarios where the $\mathrm{AV}$ is exposed to line-of-sight (LOS) GNSS signals for a short duration, such as those in urban streets. We also consider scenarios where GNSS satellites are ill-positioned causing poor geometric dilution of precision (GDOP). In these cases, non-GNSS methods are more suited and 5G is considered in this paper based on the advantages discussed above. Fig. 1 illustrates the concept of hybrid positioning using GNSS and $5 \mathrm{G}$ signals. We conduct a feasibility study on hybrid 5G-GNSS 


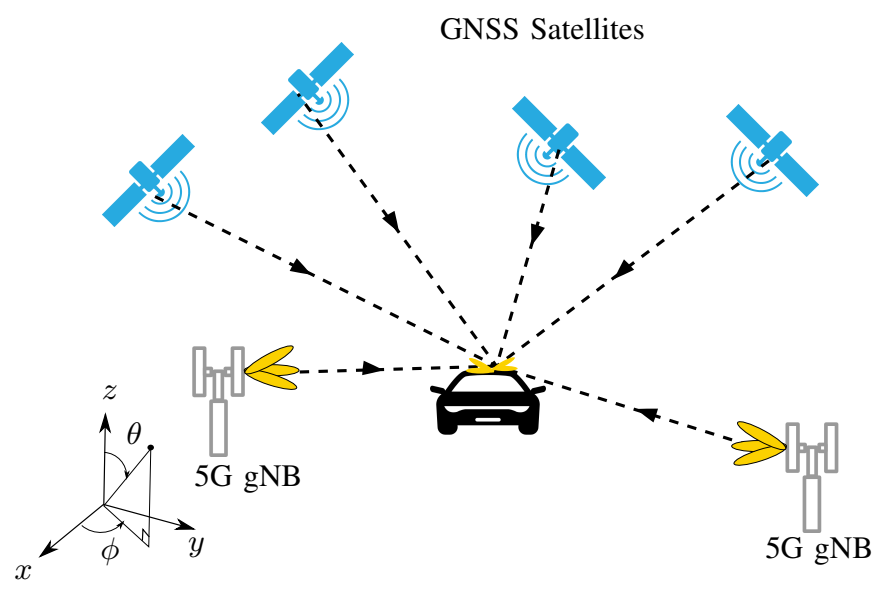

Fig. 1. A diagram illustrating the concept of hybrid positioning using GNSS and $5 \mathrm{G}$ mmWave signals.

positioning systems through the derivation of position (PEB) and velocity error bounds (VEB).

Towards that, we consider 5G mmWave transceivers with OFDM signaling. The $5 \mathrm{G}$ signal received at the $\mathrm{AV}$ is parameterized by the DOA, DOD, PR and Doppler shifts, while the signals received from GNSS satellites are parameterized by the PR and Doppler shifts. After deriving the Fisher information of the two sets of parameters, we obtain the Fisher information of position and velocity as a transformation of parameters based on geometrical relationships [11]. We then provide an analysis of when and how 5G can support GNSS, and what the parameters that govern the positioning performance are. We also provide insights and guidelines on how to design these parameters. Finally, We present a numerical investigation of the 5G performance in urban streets when GNSS has poor GDOP. Concretely, we set up a scenario comprising $5 \mathrm{G}$ base stations (gNBs) and GNSS satellites with a poor geometry due to the visibility constraints of the scenario. Ultimately, we show by simulation that the introduction of $5 \mathrm{G}$ positioning will boost the positioning availability and accuracy by a significant margin.

\section{System ModeL}

Consider an $\mathrm{AV}$ receiving downlink signals from $G$ gNBs located at $\mathbf{p}_{g}=\left[p_{g, x}, p_{g, y}, p_{g, z}\right]^{\mathrm{T}}, 1 \leq g \leq G$, and $S$ GNSS satellites located at an initial position $\mathbf{p}_{s}=\left[p_{s, x}, p_{s, y}, p_{s, z}\right]^{\mathrm{T}}$, and moving with velocities $\mathbf{v}_{s}=\left[v_{s, x}, v_{s, y}, v_{s, z}\right]^{\mathrm{T}}, 1 \leq s \leq$ $S$. The gNBs are assumed to be synchronized with the GNSS system but not with the $\mathrm{AV}$, whose bias $b_{\mathrm{u}}$ is unknown. We assume that $\mathbf{p}_{s}$ and $\mathbf{v}_{s}$ are assumed fixed during the observation time. Denote the AV's initial position, velocity and azimuth rotation angle by $\mathbf{p}=\left[p_{x}, p_{y}, p_{z}\right]^{\mathrm{T}}, \mathbf{v}=\left[v_{x}, v_{y}, v_{z}\right]^{\mathrm{T}}$ and $\phi_{0}$, respectively. Without loss of generality, we take the position of the first gNB as the origin of the coordinate system. We consider a short observation window, over which the velocities of the $\mathrm{AV}$ and satellites are assumed to be constant. This is a reasonable assumption since vehicles generally move in speeds of up to 50 or $60 \mathrm{~km} / \mathrm{h}(\approx 13.9-16.6 \mathrm{~m} / \mathrm{s})$ in the considered scenario of urban streets.

\section{A. 5G mmWave LOS OFDM Signal Model}

Consider $M$ OFDM symbols with duration $T_{0}$, including a cyclic prefix (CP) of duration $T_{\mathrm{cp}}$, sequentially transmitted over $N_{\mathrm{b}}$ beams with a carrier frequency $f_{g}$ and a subcarrier spacing $\Delta f$. The signal corresponding to the $m$-th OFDM symbol, $1 \leq m \leq M$, received over the $k$-th subcarrier, $-K / 2 \leq k \leq K / 2$, at the output of the receive beamforming is given in the frequency domain by

$$
\begin{array}{r}
\mathbf{y}_{k, m}=\sum_{g=1}^{G} \mathbf{W}_{k, m}^{(g)} \mathbf{H}_{k, m}^{(g)} \mathbf{F}_{k, m}^{(g)} \mathbf{z}_{k, m}^{(g)} \\
+\mathbf{W}_{k, m}^{(g)} \mathbf{n}_{k, m}^{(g)} \in \mathbb{C}^{N_{\mathrm{s}}},
\end{array}
$$

where $\mathbf{H}_{k, m}^{(g)} \in \mathbb{C}^{N_{\mathrm{u}} \times N_{g}}$ is the channel matrix, $\mathbf{F}_{k, m}^{(g)}=$ $\mathbf{F}_{\mathrm{RF}}^{(g)} \mathbf{F}_{\mathrm{D}_{k, m}}^{(g)} \in \mathbb{C}^{N_{g} \times N_{\mathrm{s}}}$, is the transmit beamforming matrix such that $\left\|\mathbf{F}_{k, m}^{(g)}\right\|_{\mathrm{F}}=1, \mathbf{F}_{\mathrm{RF}}^{(g)} \in \mathbb{C}^{N_{g} \times N_{\mathrm{b}}}$ and $\mathbf{F}_{\mathrm{D}_{k, m}} \in$ $\mathbb{C}^{N_{\mathrm{b}} \times N_{\mathrm{s}}}$ are the analog and digital transmit beamforming matrices, respectively. $\mathbf{z}_{k, m}^{(g)} \in \mathbb{C}^{N_{\mathrm{s}}}$ is the vector of transmitted symbols, while $N_{\mathrm{u}}$ is the number of antennas at the $\mathrm{AV}, N_{g}$ is the number of antennas at the $g$-th $\mathrm{gNB}, N_{\mathrm{b}}$ is the number of transmitted beams, and $N_{\mathrm{S}}$ is the number of transmitted symbols. The matrix $\mathbf{W}_{k, m}^{(g)}=\mathbf{W}_{\mathrm{RF}}^{(g)} \mathbf{W}_{\mathrm{D}_{k, m}}^{(g)} \in \mathbb{C}^{N_{\mathrm{u}} \times N_{\mathrm{s}}}$, such that $\left\|\mathbf{W}_{k, m}^{(g)}\right\|_{\mathrm{F}}=1, \mathbf{W}_{\mathrm{RF}}^{(g)} \in \mathbb{C}^{N_{\mathrm{u}} \times N_{\mathrm{b}}}$ and $\mathbf{W}_{\mathrm{D}_{k, m}}^{(g)} \in \mathbb{C}^{N_{\mathrm{b}} \times N_{\mathrm{s}}}$ are the analog and digital receive beamforming matrices, respectively. The additive white Gaussian noise is denoted by $\mathbf{n}_{k, m}^{(g)} \sim \mathcal{C N}\left(0, N_{0}\right) \in \mathbb{C}^{N_{\mathrm{u}}}, N_{0}$ is the noise PSD.

Remark To simplify the notation, we drop the superscript (g) from the model in (1). The signal corresponding to a specific $\mathrm{gNB}$ can be therefore obtained using the parameters related to that $g N B$. The subscript $\mathrm{g}$ is used to distinguish the parameters related to gNBs from those related to the $A V$ or GNSS satellites. This is similar to consider only one $g N B$, and treating different $g N B$ s independently.

Based on this remark, the definition of the notation in (1) is now explained. The channel matrix is given by

$$
\mathbf{H}_{k, m}=\kappa_{k, m} \mathbf{a}_{\mathrm{u}, k}\left(\theta_{\mathrm{u}}, \phi_{\mathrm{u}}\right) \mathbf{a}_{\mathrm{g}, k}^{\mathrm{H}}\left(\theta_{\mathrm{g}}, \phi_{\mathrm{g}}\right),
$$

where

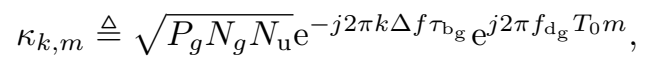

such that $\left|\kappa_{k, m}\right|^{2}=P_{g} N_{g} N_{\mathrm{u}}$, and $P_{g}$ is the average received power from the gNB. The complex channel gain is assumed to have been compensated for. $\tau_{\mathrm{bg}_{\mathrm{g}}}$ and $f_{\mathrm{dg}}$ are biased $\mathrm{TOA}^{1}$ and Doppler frequency. All the OFDM symbols are assumed to be delayed by the same TOA. $\left(\theta_{\mathrm{u}}, \phi_{\mathrm{u}}\right)$ and $\left(\theta_{\mathrm{g}}, \phi_{\mathrm{g}}\right)$ are the DOA and DOD, respectively. $\mathbf{a}_{\mathrm{u}, k}\left(\theta_{\mathrm{u}}, \phi_{\mathrm{u}}\right)$ is the array response vectors of the $\mathrm{AV}$ defined by

$$
\mathbf{a}_{\mathrm{u}, k}\left(\theta_{\mathrm{u}}, \phi_{\mathrm{u}}\right)=\frac{1}{\sqrt{N_{\mathrm{u}}}} \mathrm{e}^{-j \frac{2 \pi}{\lambda_{k}} \mathbf{L}_{\mathrm{u}} \mathbf{u}\left(\theta_{\mathrm{u}}, \phi_{\mathrm{u}}\right)}
$$

\footnotetext{
${ }^{1}$ Consider a transmitted signal $\mathbf{g}(t)$, where $t$ is taken with reference to the gNB's clock. The signal is received as $\mathbf{g}(t-\tau+v t / c)$ where $\tau$ is the propagation delay and $v$ is the relative speed of motion. To write the received signal with reference to the AV's clock that is biased by $b_{\mathrm{u}}$ with respect to the $\mathrm{gNB}$, replace $t$ by $t-b_{\mathrm{u}}$ and use $f_{\mathrm{d}_{\mathrm{g}}} / f_{g}=v / c$ to obtain $\mathbf{g}\left(\left(1+f_{\mathrm{dg}_{\mathrm{g}}} / f_{g}\right)(t-\right.$ $\left.\left.b_{\mathrm{u}}\right)-\tau\right)$. That is, the biased TOA $\tau_{\mathrm{b}_{\mathrm{g}}}=\left(1+f_{\mathrm{d}_{\mathrm{g}}} / f_{g}\right) b_{\mathrm{u}}+\tau \approx b_{\mathrm{u}}+\tau$.
} 
$\mathbf{L}_{\mathrm{u}} \in \mathbb{R}^{N_{\mathrm{u}} \times 3}$ is the antenna location matrix in half-wavelength, $\lambda_{k}=\frac{c}{f_{g}+k \Delta f}$ and $\mathbf{u}(\theta, \phi)=[\cos \phi \sin \theta, \sin \phi \sin \theta, \cos \theta]^{\mathrm{T}}$ is a unit vector pointing towards an azimuth angle $\phi$ and an elevation angle $\theta . \mathbf{a}_{\mathrm{g}, k}\left(\theta_{\mathrm{g}}, \phi_{\mathrm{g}}\right)$ can be defined similarly. Note that in this model, the complex channel gain assumed to be estimated a priori.

Finally, note that Doppler shift introduces a frequency error that may cause loss of sub-carrier orthogonality. Therefore, $\mathbf{z}_{k, m} \in \mathbb{C}^{N_{\mathrm{s}}}$ is the signal transmitted on the $k$-th subcarrier including the interference from adjacent subcarriers and is modeled by [12]

$$
\mathbf{z}_{k, m}=\mathbf{x}_{k, m}+\sum_{k^{\prime} \neq k} \mathbf{x}_{k^{\prime}, m} c_{k-k^{\prime}}\left(f_{\mathrm{d}_{\mathrm{g}}} T_{\mathrm{s}}\right)=\mathbf{X}_{m} \mathbf{c}_{k},
$$

where $\mathbf{X}_{m} \triangleq\left[\mathbf{x}_{-\frac{K}{2}, m}, \cdots, \mathbf{x}_{\frac{K}{2}, m}\right]$ such that $\mathbf{x}_{k, m} \triangleq$ $\left[X_{k, m}^{(1)}, \cdots, X_{k, m}^{\left(N_{\mathrm{s}}\right)}\right]^{\mathrm{T}}$ is the vector of $N_{\mathrm{s}}$ transmitted symbols, with a duration $T_{\mathrm{s}}$, and $\mathbf{c}_{k}$ is the $(k+1+K / 2)$-th column of the circulant matrix $\mathbf{C}=j 2 \pi f_{\mathrm{d}_{\mathrm{g}}} T_{s} \mathbf{D}^{\mathrm{H}} \mathbf{Q D}+\mathbf{I}_{K}$, where $\mathbf{Q}=\operatorname{diag}(-K / 2,-K / 2+1, \cdots, K / 2)$ and $\mathbf{D}$ is the DFT matrix [12].

\section{B. GNSS Satellite Signals}

Assuming that the Doppler frequency is much less than the carrier frequency ${ }^{2}$, the signal received from the $s$-th GNSS satellite, $1 \leq s \leq S$, can be written as

$$
y_{s}(t)=\sqrt{P_{\mathrm{s}}} x_{s}\left(t-\tau_{\mathrm{b}_{s}}\right) \mathrm{e}^{j 2 \pi f_{d_{s}} t}+n_{s}(t),
$$

where $P_{\mathrm{s}}, f_{\mathrm{d}_{s}}, \tau_{\mathrm{b}_{s}}$ and $\varphi_{\mathrm{s}}$ are the received power, Doppler frequency and biased TOA and phase delay, respectively. $x_{s}(t)$ is the reference signal transmitted from the $s$-th satellite and modeled as

$$
x_{s}(t)=\sum_{\ell=0}^{N_{\mathrm{so}}-1} c_{s \ell} r\left(t-\ell T_{\mathrm{c}}\right)
$$

where $c_{s \ell}$ is the $\ell$-th PN-code chip with duration $T_{c}, r(t)$ is the pulse-shaping filter and $N_{\text {so }}$ is the total number of transmitted chips.

\section{PEB AND VEB DERIVATION}

\section{A. Derivation of GNSS and $5 G$ FIMs}

The vectors of unknowns associated with the $g$-th gNB signal and the $s$-th satellite can be written as

$$
\begin{aligned}
& \boldsymbol{\eta}_{\mathrm{g}} \triangleq\left[\theta_{\mathrm{g}}, \phi_{\mathrm{g}}, \theta_{\mathrm{u}}, \phi_{\mathrm{u}}, \tau_{\mathrm{b}_{\mathrm{g}}}, f_{\mathrm{d}_{\mathrm{g}}}\right]^{\mathrm{T}} \in \mathbb{R}^{6}, \\
& \boldsymbol{\eta}_{\mathrm{s}} \triangleq\left[\tau_{\mathrm{b}_{\mathrm{s}}}, f_{\mathrm{d}_{\mathrm{s}}}\right]^{\mathrm{T}} \in \mathbb{R}^{2} .
\end{aligned}
$$

From (1), defining $\boldsymbol{\mu}_{k, m} \triangleq \mathbf{W}_{k, m}^{\mathrm{H}} \mathbf{H}_{k, m} \mathbf{F}_{k, m} \mathbf{z}_{k, m}$, then $\mathbf{J}_{\mathrm{g}} \in$ $\mathbb{R}^{6 \times 6}$, the Fisher information matrix of $\boldsymbol{\eta}_{\mathrm{g}}$, can be computed element-wise using [11]

$$
\left[\mathbf{J}_{\mathrm{g}}\right]_{a, b}=\frac{1}{N_{0}} \sum_{\forall k, m} \Re\left\{\frac{\partial \boldsymbol{\mu}_{k, m}^{\mathrm{H}}}{\partial \eta_{\mathrm{g}}}\left(\mathbf{W}_{k, m}^{\mathrm{H}} \mathbf{W}_{k, m}\right)^{-1} \frac{\partial \boldsymbol{\mu}_{k, m}}{\partial \eta_{\mathrm{g}}}\right\},
$$

where $\eta_{\mathrm{g}}$ is the $a$-th element in $\boldsymbol{\eta}_{\mathrm{g}}, 1 \leq a, b \leq 6$. The derivation of the elements of $\mathbf{J}_{\mathrm{g}}$ is provided in Appendix A.

\footnotetext{
${ }^{2}$ This assumption enables us write the signal using $t$ instead of $\left(1+\frac{f_{d_{s}}}{f_{s}}\right) t$
}

Note how $\mathbf{z}_{k, m}$ depends on $f_{\mathrm{d}_{\mathrm{g}}}$, which is accounted for when the FIM is derived. See (16f).

Similarly, defining $\mu_{s}(t)=\sqrt{P_{\mathrm{s}}} x_{s}\left(t-\tau_{\mathrm{b}_{s}}\right) \mathrm{e}^{j 2 \pi f_{d_{s}} t}$ from (6), then $\mathbf{J}_{s} \in \mathbb{R}^{2 \times 2}$, the Fisher information matrix of $\boldsymbol{\eta}_{\mathrm{s}}$ is given by

$$
\left[\mathbf{J}_{s}\right]_{a, b}=\frac{1}{N_{0}} \int_{0}^{T_{\mathrm{so}}} \Re\left\{\frac{\partial \mu_{s}^{*}(t)}{\partial \eta_{\mathrm{s}}} \frac{\partial \mu_{s}(t)}{\partial \eta_{\mathrm{g}}}\right\} \mathrm{d} t,
$$

where $T_{\mathrm{so}}=N_{\mathrm{so}} T_{\mathrm{c}}$ is the satellite signal observation time and $1 \leq a, b \leq 2$. Note that we assume observations from different satellites to be independent. As shown in Appendix B

$$
\mathbf{J}_{s}=\frac{4 \pi^{2} P_{\mathrm{s}} T_{\mathrm{so}}}{N_{0}}\left[\begin{array}{cc}
W_{\mathrm{eff}}^{2} & 0 \\
0 & T_{\mathrm{eff}}^{2}
\end{array}\right]
$$

where

$$
\begin{aligned}
& W_{\mathrm{eff}}^{2} \triangleq \frac{1}{T_{\mathrm{c}}} \int_{-W / 2}^{W / 2} f^{2}|R(f)|^{2} \mathrm{~d} f \\
& T_{\mathrm{eff}}^{2} \triangleq \int_{0}^{T_{\mathrm{c}}} \vec{t}^{2}|r(t)|^{2} \mathrm{~d} t
\end{aligned}
$$

$\bar{t}^{2} \triangleq \frac{1}{N_{\mathrm{so}}} \sum_{\ell=0}^{N_{\mathrm{so}}-1}\left(t+\ell T_{\mathrm{c}}\right)^{2}$ and $R(f)$ is the PSD of $r(t)$, assumed to be symmetric around $f=0$.

\section{B. Position and Velocity Error Bounds}

We are interested in the AV position $\mathbf{p}$, velocity $\mathbf{v}$ and clock bias $b_{\mathrm{u}}$, and consequently need to compute the FIM of

$$
\boldsymbol{\eta}^{\prime} \triangleq\left[\mathbf{p}^{\mathrm{T}}, \mathbf{v}^{\mathrm{T}}, b_{\mathrm{u}}\right]^{\mathrm{T}} \in \mathbb{R}^{7}
$$

as a transformation of parameters. Given that $\mathbf{J}_{\mathrm{g}}$ and $\mathbf{J}_{\mathrm{s}}$ provide independent information, they can be transformed separately as

$$
\mathbf{J}_{\boldsymbol{\eta}^{\prime}}=\underbrace{\sum_{g=1}^{G} \mathbf{T}_{\mathrm{g}} \mathbf{J}_{\mathrm{g}} \mathbf{T}_{\mathrm{g}}^{\mathrm{T}}}_{\text {Information from } 5 \mathrm{G}}+\underbrace{\sum_{s=1}^{S} \mathbf{T}_{s} \mathbf{J}_{s} \mathbf{T}_{s}^{\mathrm{T}}}_{\text {Information from GNSS }} \in \mathbb{R}^{7 \times 7},
$$

where $\mathbf{T}_{\mathrm{g}} \triangleq \frac{\partial \boldsymbol{\eta}_{\mathrm{g}}^{\mathrm{T}}}{\partial \boldsymbol{\eta}^{\prime}} \in \mathbb{R}^{7 \times 6}$ and $\mathbf{T}_{s} \triangleq \frac{\partial \boldsymbol{\eta}_{s}^{\mathrm{T}}}{\partial \boldsymbol{\eta}^{\prime}} \in \mathbb{R}^{7 \times 2}$, obtained in Appendix $\mathrm{C}$ using the following formulas:

$$
\begin{aligned}
\theta_{\mathrm{g}} & =\cos ^{-1}\left(\frac{p_{z}-p_{g, z}}{\left\|\mathbf{p}-\mathbf{p}_{g}\right\|}\right), \\
\phi_{\mathrm{g}} & =\tan ^{-1}\left(\frac{p_{y}-p_{g, y}}{p_{x}-p_{g, x}}\right), \\
\theta_{\mathrm{u}} & =\cos ^{-1}\left(\frac{-p_{z}+p_{g, z}}{\left\|\mathbf{p}-\mathbf{p}_{g}\right\|}\right), \\
\phi_{\mathrm{u}} & =\tan ^{-1}\left(\frac{p_{y}-p_{g, y}}{p_{x}-p_{g, x}}\right)-\phi_{0}-\pi \\
f_{\mathrm{d}_{\mathrm{g}}} & =-\frac{\left(\mathbf{v}-\mathbf{v}_{g}\right)^{\mathrm{T}}\left(\mathbf{p}-\mathbf{p}_{g}\right)}{\lambda_{g}\left\|\mathbf{p}-\mathbf{p}_{\mathrm{g}}\right\|}, \\
\tau_{\mathrm{b}_{\mathrm{g}}} & =b_{\mathrm{u}}+\frac{\left\|\mathbf{p}-\mathbf{p}_{\mathrm{g}}\right\|}{c}, \\
f_{\mathrm{d}_{s}} & =-\frac{\left(\mathbf{v}-\mathbf{v}_{s}\right)^{\mathrm{T}}\left(\mathbf{p}-\mathbf{p}_{s}\right)}{\lambda_{s}\left\|\mathbf{p}-\mathbf{p}_{s}\right\|}, \\
\tau_{\mathrm{b} s} & =b_{\mathrm{u}}+\frac{\left\|\mathbf{p}-\mathbf{p}_{s}\right\|}{c} .
\end{aligned}
$$


where $c=f_{g} \lambda_{g}=f_{\mathrm{s}} \lambda_{\mathrm{s}}$ is the speed of light. Note that for each $\mathrm{gNB}, \mathbf{J}_{\mathrm{g}}$ and $\mathbf{T}_{\mathrm{g}}$ in (14) are computed from (10) and (24), respectively, using the parameters of that gNB.

Finally, we obtain $\mathbf{J}_{\mathbf{p}, \mathbf{v}}^{\mathrm{e}}$, the EFIM of $\mathbf{p}$ and $\mathbf{v}$, by writing $\mathbf{J}_{\eta^{\prime}}$ in block form as

$$
\mathbf{J}_{\boldsymbol{\eta}^{\prime}}=\left[\begin{array}{cc}
\mathbf{J}_{\mathbf{p}, \mathbf{v}} & \mathbf{J}_{\mathbf{p v}, b_{\mathrm{u}}} \\
\mathbf{J}_{\mathbf{p v}, b_{\mathrm{u}}}^{\mathrm{T}} & J_{b_{\mathrm{u}}}
\end{array}\right] .
$$

Using Schur complement, we can derive

$$
\mathbf{J}_{\mathbf{p}, \mathbf{v}}^{\mathrm{e}}=\mathbf{J}_{\mathbf{p}, \mathbf{v}}-\frac{1}{J_{b_{\mathbf{u}}}} \mathbf{J}_{\mathbf{p v}, b_{\mathbf{u}}} \mathbf{J}_{\mathbf{p v}, b_{\mathrm{u}}}^{\mathrm{T}} \in \mathbb{R}^{6 \times 6}
$$

Consequently, defining $\mathbf{c}=\operatorname{diag}\left\{\left(\mathbf{J}_{\mathbf{p}, \mathbf{v}}^{\mathbf{e}}\right)^{-1}\right\}$, then

$$
\begin{aligned}
\mathrm{PEB} & \triangleq \sqrt{c_{1}+c_{2}+c_{3}}, \\
\mathrm{VEB} & \triangleq \sqrt{c_{4}+c_{5}+c_{6}} .
\end{aligned}
$$

Note that for the position and velocity to be computed with no ambiguity, $\mathbf{J}_{\eta^{\prime}}$ must be rank 7 . Since $\mathbf{J}_{\mathrm{g}}$ is rank 6 , at least $2 \mathrm{gNBs}$ are needed to obtain PEB and VEB based on $5 \mathrm{G}$ only, when the clock bias is unknown. Similarly, $\mathbf{J}_{\mathrm{s}}$ is rank 2, which leads to the widely known fact that at least 4 satellites are needed to compute the position and velocity in GNSS positioning systems. In principle, hybridization allows us to use less than this number of satellites and $\mathrm{gNBs}$, as we can use, for example, $1 \mathrm{gNB}$ and 1 satellite. The satellite signal can be used to estimate the AV clock bias, while the gNB signals can be used to estimate the position and velocity. It is intuitive that incorporating more signals can boost the performance.

\section{NumericAl Results}

\section{A. Geometry}

Two scenarios related to different satellite arrangements are presented in this section: an open visibility scenario, and a constrained visibility scenario:

- Scenario A: Open Visibility This is a reference scenario where the AV receives LOS signals from 4 GNSS satellites observed at well-spaced locations. These locations are given in spherical coordinates (See Fig. 1) as $\mathbf{p}_{s}=(\rho, \theta, \phi)$, where $\rho=20.2 \times 10^{6}$ and $(\theta, \phi)=\left(35.2^{\circ}, 45^{\circ}\right),\left(35.2^{\circ},-135^{\circ}\right),\left(57.3^{\circ}, 130^{\circ}\right)$ and $\left(57.37^{\circ},-39.8^{\circ}\right)$, respectively.

- Scenario B: Constrained Visibility In this visibilityconstrained scenario, the AV receives LOS signals from 4 GNSS satellites that are almost aligned on an arc, that is, a narrow azimuth sector. Such a scenario can arise in central business districts and other suburban areas where high-rising buildings limit the duration and the visibility of LOS satellite links, causing positioning to be challenging. The 4 satellites are assumed to be located at $\mathbf{p}_{s}=(\rho, \theta, \phi)$, where $\rho=20.2 \times 10^{6}$ and $(\theta, \phi)=\left(45^{\circ}, 0.08^{\circ}\right),\left(5^{\circ},-0.66^{\circ}\right),\left(17^{\circ}, 0.20^{\circ}\right)$ and $\left(25^{\circ},-0.14^{\circ}\right)$, respectively.

The satellites are assumed to move at a speed of $3.9 \mathrm{~km} / \mathrm{s}$ [13], of which a maximum of $1 \mathrm{~km} / \mathrm{s}$ is in the radial direction.

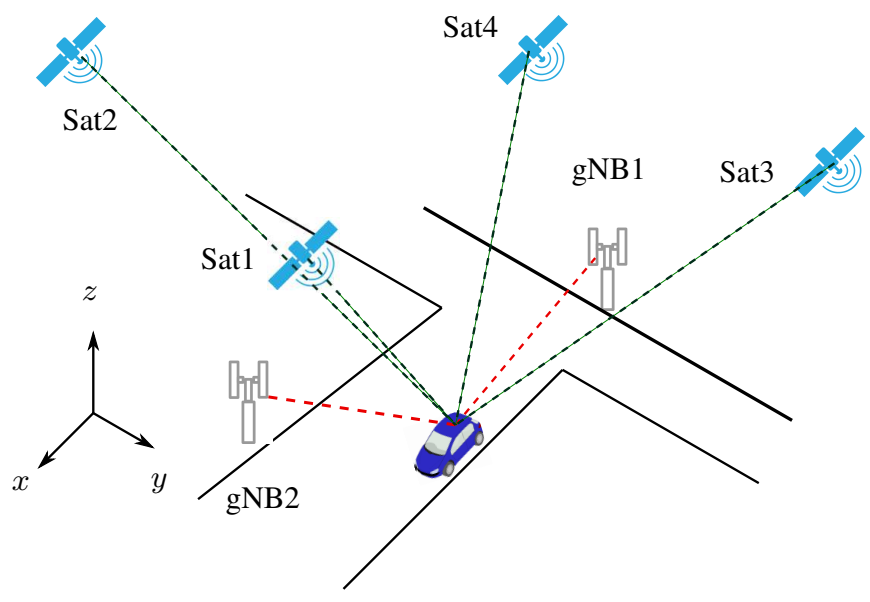

Fig. 2. Scenario A: Four GNSS satellites observed at well-spaced locations.

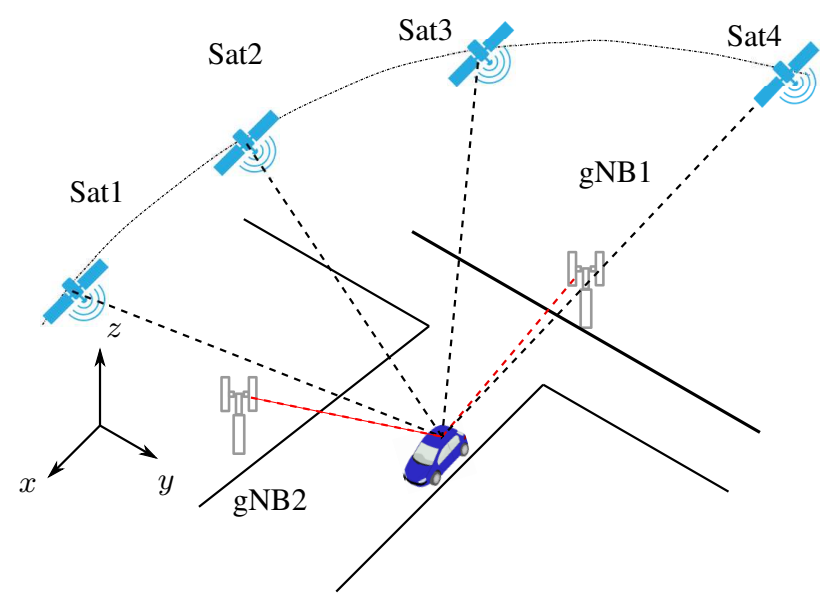

Fig. 3. Scenario B: Four GNSS satellites with poor GDOP, resulting from the narrow azimuth sector observed.

The tangential direction is chosen arbitrarily in the plane orthogonal to the radial direction.

In both scenarios, we consider two gNBs, each equipped with an $12 \times 12$ uniform rectangular array, centered at $[0,0,7]^{\mathrm{T}}$ $\mathrm{m}$ and $[20,-6,5]^{\mathrm{T}} \mathrm{m}$ oriented towards the $+x$-axis and the $+y$-axis, respectively. Note that the origin of the coordinate systems is arbitrarily chosen to be on the ground under the first gNB. The AV, equipped with an $8 \times 8$ array oriented toward the $+z$-axis, is assumed to be moving along the $x$-axis with a speed of $50 \mathrm{~km} / \mathrm{h}$ and measurements are taken when the AV is at $p_{x}=10 \mathrm{~m}$.

\section{B. Transceiver Parameters}

For the OFDM signals, we consider $K=1024$ subcarriers and $M=1000$ symbols transmitted over a carrier frequency $38 \mathrm{GHz}$ and bandwidth of $125 \mathrm{MHz}$. That is, the observation time is $8.2 \mathrm{~ms}$. The pilot samples are generated randomly as complex normal vectors such that $\left\|\mathbf{x}_{m, k}\right\|^{2}=1$. The nonorthogonality of the subcarriers occurring due to the Doppler shift is assumed to affect one adjacent subcarrier on each side. 


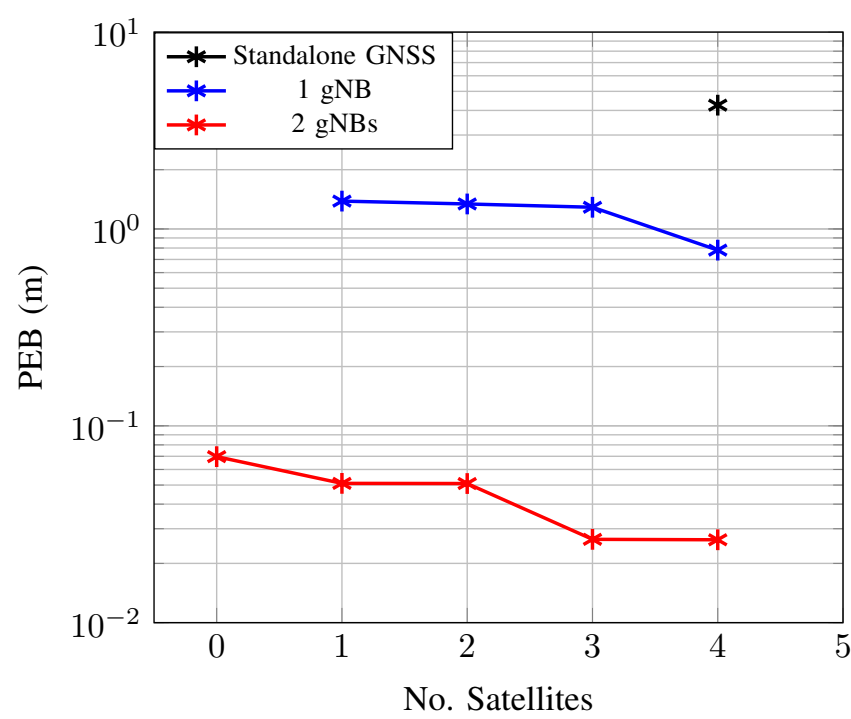

Fig. 4. PEB under Scenario A comprising well-spaced GNSS satellites.

That is, the sum in (5) is taken for $k^{\prime}=k \pm 1$. Finally, $P_{g} / N_{0}=30 \mathrm{dBHz}$ [14].

We consider L1 GNSS signals with a carrier frequency of 1575.42 MHz and a bandwidth of $1.023 \mathrm{MHz}$. The signals are received with a carrier-to-noise ratio of $P_{s} / N_{0}=40 \mathrm{dBHz}$ for a duration of $300 \mathrm{~ms}$. The observation time and bandwidth of both systems are summarized in Table I.

\section{Position Error Bounds}

Fig. 4 illustrates the PEB under various cases with wellpositioned satellites as per Scenario A, defined in Section IV-A. With reference to the discussion below (15a), hybrid positioning requires at least $1 \mathrm{gNB}$ and 1 satellite, which is providing a PEB of $1.4 \mathrm{~m}$ in the studied scenario. The standalone GNSS PEB $(4.25 \mathrm{~m})$ represents the poorest case among those studied in Fig. 4, but adding a single gNB brings PEB down to $75 \mathrm{~cm}$. Adding a second gNB leads to further performance enhancement with a $\mathrm{PEB}$ of $2.5 \mathrm{~cm}$.

The performance under Scenario B, whereby satellite locations cause poor GDOP is shown in Fig. 5. Not that with the exception of the standalone GNSS PEB, Fig. 5 shows that hybrid positioning in Scenario B provides a performance comparable to that under Scenario A. This implies that the performance of hybrid positioning is mainly governed by the abundant resources provided by $5 \mathrm{G}$ gNBS, as can be seen in Table I.

\section{Velocity Error Bounds}

From Fig. 6, it can be seen that standalone GNSS system can provides a highly accurate velocity estimate, thanks to the long observation time and the good geometrical location of the satellites in Scenario A. However, although 5G provides a less accurate velocity estimate, it is in the order of a few centimeters, which is acceptable in systems of AVs.

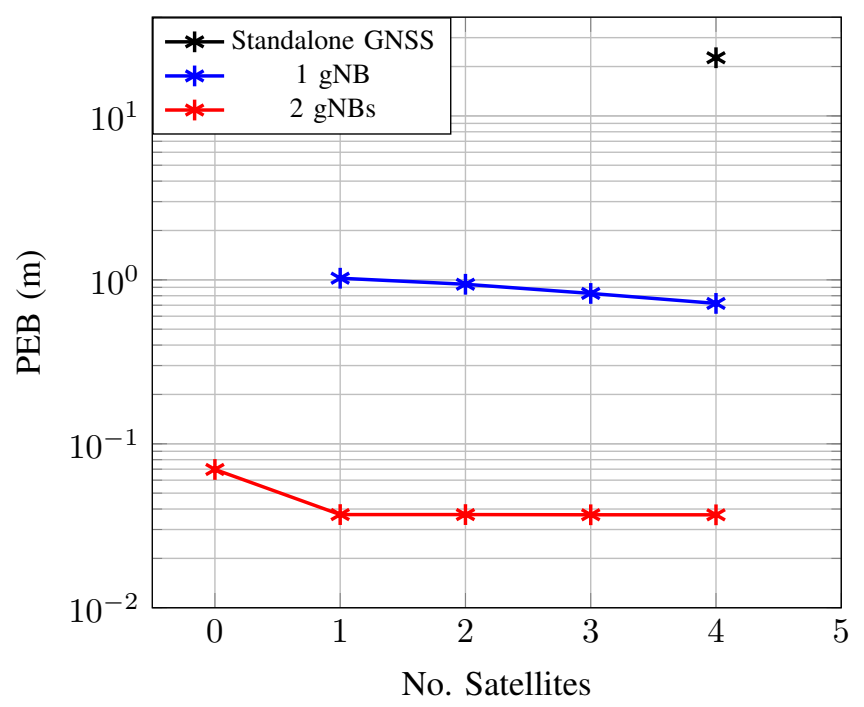

Fig. 5. PEB under Scenario B comprising GNSS satellites with poor GDOP.

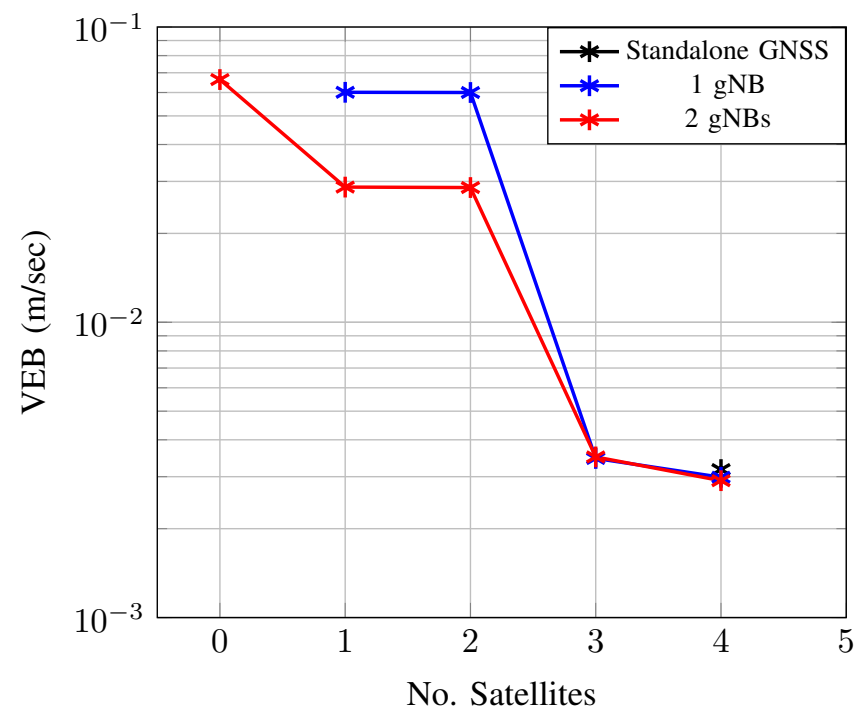

Fig. 6. VEB under Scenario A comprising well-spaced GNSS satellites.

Considering Fig. 7, it can be seen that when access to two gNBs or 4 satellites is not available, hybridization under illarranged satellites can be useful when access to only one gNB and 2-3 satellites is possible. Under the latter case, it is possible to obtain a VEB of $1.5-1.8 \mathrm{~m} / \mathrm{sec}$.

\section{CONCLUSIONS}

This paper investigated the benefits of integrating $5 \mathrm{G}$ positioning with GNSS positioning. We presented theoretical results by deriving the position (PEB) and velocity (VEB) error bounds, and numerical results focusing on two possible scenarios whereby the satellites are well- and ill-positioned. Our numerical results show that hybridization of these two systems is beneficial when both fail individually. it was also evident from our numerical results that when simultaneous access to $2 \mathrm{gNBs}$ is available, precedence should be given 
TABLE I

OBSERVATION TIME AND BANDWIDTH OF GNSS AND 5G SYSTEMS.

\begin{tabular}{|c|c|c|c|c|}
\cline { 2 - 5 } \multicolumn{1}{c|}{} & $\begin{array}{c}\text { Observation Time } \\
(\mathrm{ms})\end{array}$ & $\begin{array}{c}\text { Bandwidth } \\
(\mathrm{MHz})\end{array}$ & $\begin{array}{c}P / N_{0} \\
(\mathrm{dBHz})\end{array}$ & $\begin{array}{c}\text { Total Energy= } \\
\text { Time } \times \text { Bandwidth } \times P / N_{0}\end{array}$ \\
\hline GNSS & 300 & 1.023 & 40 & $307 \times 10^{7}$ \\
\hline $5 \mathrm{G}$ & 8.2 & 125 & 30 & $102.5 \times 10^{7}$ \\
\hline
\end{tabular}

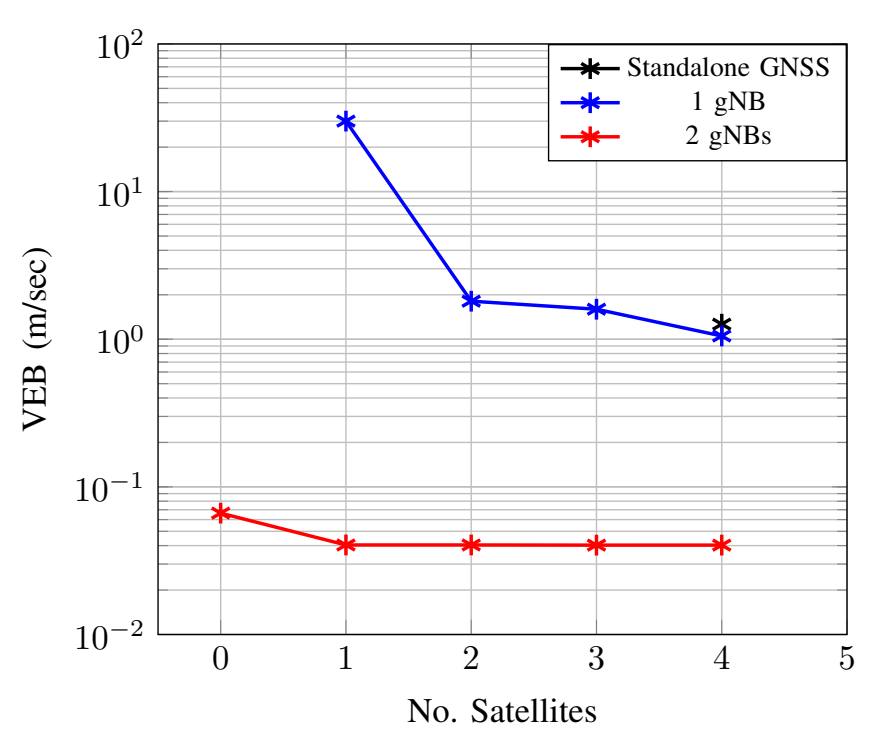

Fig. 7. VEB under Scenario B comprising GNSS satellites with poor GDOP.

to $5 \mathrm{G}$ positioning as it seems to provide satisfactory $\mathrm{PEB}$ and VEB. This can be attributed to the fact that the time-bandwidth resources available under $5 \mathrm{G}$ are more than triple of those available under GNSS (See Table I).

\section{APPENDIX A}

FISHER INFORMATION MATRIX OF 5G PARAMETERS

We drop the angle parameters of $\mathbf{a}_{\mathrm{u}, k}$ and $\mathbf{a}_{\mathrm{g}, k}$ to simplify the notation. Then, defining

$$
\begin{array}{ll}
\dot{\mathbf{a}}_{\mathrm{g}, \theta, k} \triangleq \frac{\partial \mathbf{a}_{\mathrm{g}, k}}{\partial \theta_{\mathrm{g}}}, & \dot{\mathbf{a}}_{\mathrm{g}, \phi, k} \triangleq \frac{\partial \mathbf{a}_{\mathrm{g}, k}}{\partial \phi_{\mathrm{g}}}, \\
\dot{\mathbf{a}}_{\mathrm{u}, \theta, k} \triangleq \frac{\partial \mathbf{a}_{\mathrm{u}, k}}{\partial \theta_{\mathrm{u}}}, & \dot{\mathbf{a}}_{\mathrm{u}, \phi, k} \triangleq \frac{\partial \mathbf{a}_{\mathrm{u}, k}}{\partial \phi_{\mathrm{u}}},
\end{array}
$$

and noting that $\dot{\mathbf{z}}_{f_{\mathrm{dg}}, k, m} \triangleq \frac{\partial \mathbf{z}_{\mathrm{u}, k}}{\partial f_{\mathrm{dg}}}=\mathbf{X}_{m} \dot{\mathbf{c}}_{k}$, where $\dot{\mathbf{c}}_{k}$ is the $\left(k+\frac{K}{2}+1\right)$-th column of $j 2 \pi T_{s} \mathbf{D}^{\mathrm{H}} \mathbf{Q D}$, we derive

$$
\begin{aligned}
\frac{\partial \boldsymbol{\mu}_{k, m}}{\partial \theta_{\mathrm{g}}} & =\kappa_{k, m} \mathbf{W}_{k, m}^{\mathrm{H}} \mathbf{a}_{\mathrm{u}, k} \dot{\mathbf{a}}_{\mathrm{g}, \theta, k}^{\mathrm{H}} \mathbf{F}_{k, m} \mathbf{z}_{k, m}, \\
\frac{\partial \boldsymbol{\mu}_{k, m}}{\partial \phi_{\mathrm{g}}} & =\kappa_{k, m} \mathbf{W}_{k, m}^{\mathrm{H}} \mathbf{a}_{\mathrm{u}, k} \dot{\mathbf{a}}_{\mathrm{g}, \phi, k}^{\mathrm{H}} \mathbf{F}_{k, m} \mathbf{z}_{k, m}, \\
\frac{\partial \boldsymbol{\mu}_{k, m}}{\partial \theta_{\mathrm{u}}} & =\kappa_{k, m} \mathbf{W}_{k, m}^{\mathrm{H}} \dot{\mathbf{a}}_{\mathrm{u}, \theta, k} \mathbf{a}_{\mathrm{g}, k}^{\mathrm{H}} \mathbf{F}_{k, m} \mathbf{z}_{k, m}, \\
\frac{\partial \boldsymbol{\mu}_{k, m}}{\partial \phi_{\mathrm{u}}} & =\kappa_{k, m} \mathbf{W}_{k, m}^{\mathrm{H}} \dot{\mathbf{a}}_{\mathrm{u}, \phi, k} \mathbf{a}_{\mathrm{g}, k}^{\mathrm{H}} \mathbf{F}_{k, m} \mathbf{z}_{k, m}, \\
\frac{\partial \boldsymbol{\mu}_{k, m}}{\partial \tau_{\mathrm{b}}} & =(-j 2 \pi k \Delta f) \kappa_{k, m} \mathbf{W}_{k, m}^{\mathrm{H}} \mathbf{a}_{\mathrm{u}, k} \mathbf{a}_{\mathrm{g}, k}^{\mathrm{H}} \mathbf{F}_{k, m} \mathbf{z}_{k, m},
\end{aligned}
$$

$$
\begin{aligned}
\frac{\partial \boldsymbol{\mu}_{k, m}}{\partial f_{\mathrm{d}_{\mathrm{g}}}}= & \kappa_{k, m} \mathbf{W}_{k, m}^{\mathrm{H}} \mathbf{a}_{\mathrm{u}, k} \mathbf{a}_{\mathrm{g}, k}^{\mathrm{H}} \mathbf{F}_{k, m} \times \\
& \left(\left(j 2 \pi T_{0} m\right) \mathbf{z}_{k, m}+\dot{\mathbf{z}}_{f_{\mathrm{dg}}, k, m}\right) .
\end{aligned}
$$

For compactness, we also introduce the following notation

$$
\begin{aligned}
\gamma_{0} & \triangleq \frac{P_{g} N_{g} N_{\mathrm{u}}}{N_{0}}, \\
\alpha, & \triangleq-j 2 \pi \Delta f, \\
\breve{\mathbf{F}}_{k, m} & =\mathbf{F}_{k, m} \mathbf{z}_{k, m} \mathbf{z}_{k, m}^{\mathrm{H}} \mathbf{F}_{k, m}^{\mathrm{H}}, \\
\breve{\mathbf{F}}_{k, m} & =\mathbf{F}_{k, m} \dot{\mathbf{z}}_{k, m} \mathbf{z}_{k, m}^{\mathrm{H}} \mathbf{F}_{k, m}^{\mathrm{H}}, \\
\breve{\mathbf{F}}_{k, m} & =\mathbf{F}_{k, m} \dot{\mathbf{z}}_{k, m} \dot{\mathbf{z}}_{k, m}^{\mathrm{H}} \mathbf{F}_{k, m}^{\mathrm{H}}, \\
\breve{\mathbf{W}}_{k, m} & =\mathbf{W}_{k, m}\left(\mathbf{W}_{k, m}^{\mathrm{H}} \mathbf{W}_{k, m}\right)^{-1} \mathbf{W}_{k, m}^{\mathrm{H}}, \\
\dot{\mathbf{G}}_{k, m} & \triangleq j 2 \pi T_{0} m \breve{\mathbf{F}}_{k, m}+\breve{\mathbf{F}}_{k, m}, \\
\ddot{\mathbf{G}}_{k, m} & \triangleq\left(2 \pi T_{0} m\right)^{2} \breve{\mathbf{F}}_{k, m}-4 \pi T_{0} m \Im\left\{\breve{\mathbf{F}}_{k, m}\right\}+\breve{\mathbf{F}}_{k, m} .
\end{aligned}
$$

Next, the elements of (10) can be obtained using (16) as

$$
\begin{aligned}
& J_{\theta_{\mathrm{g}}}=\gamma_{0} \sum_{\forall k, m} \Re\left\{\left(\dot{\mathbf{a}}_{\mathrm{g}, \theta, k}^{\mathrm{H}} \breve{\mathbf{F}}_{k, m} \dot{\mathbf{a}}_{\mathrm{g}, \theta, k}\right)\left(\mathbf{a}_{\mathrm{u}, k}^{\mathrm{H}} \breve{\mathbf{W}}_{k, m} \mathbf{a}_{\mathrm{u}, k}\right)\right\}, \\
& J_{\theta_{\mathrm{g}} \phi_{\mathrm{g}}}=\gamma_{0} \sum_{\forall k, m} \Re\left\{\left(\dot{\mathbf{a}}_{\mathrm{g}, \phi, k}^{\mathrm{H}} \breve{\mathbf{F}}_{k, m} \dot{\mathbf{a}}_{\mathrm{g}, \theta, k}^{\mathrm{H}}\right)\left(\mathbf{a}_{\mathrm{u}, k}^{\mathrm{H}} \breve{\mathbf{W}}_{k, m} \mathbf{a}_{\mathrm{u}, k}\right)\right\}, \\
& J_{\theta_{\mathrm{g}} \theta_{\mathrm{u}}}=\gamma_{0} \sum_{\forall k, m} \Re\left\{\left(\mathbf{a}_{\mathrm{g}, k}^{\mathrm{H}} \breve{\mathbf{F}}_{k, m} \dot{\mathbf{a}}_{\mathrm{g}, \theta, k}^{\mathrm{H}}\right)\left(\mathbf{a}_{\mathrm{u}, k}^{\mathrm{H}} \breve{\mathbf{W}}_{k, m} \dot{\mathbf{a}}_{\mathrm{u}, \theta, k}^{\mathrm{H}}\right)\right\}, \\
& J_{\theta_{\mathrm{g}} \phi_{\mathrm{u}}}=\gamma_{0} \sum_{\forall k, m} \Re\left\{\left(\mathbf{a}_{\mathrm{g}, k}^{\mathrm{H}} \breve{\mathbf{F}}_{k, m} \dot{\mathbf{a}}_{\mathrm{g}, \theta, k}^{\mathrm{H}}\right)\left(\mathbf{a}_{\mathrm{u}, k}^{\mathrm{H}} \breve{\mathbf{W}}_{k, m} \dot{\mathbf{a}}_{\mathrm{u}, \phi, k}^{\mathrm{H}}\right)\right\}, \\
& J_{\theta_{\mathrm{g}} \tau_{\mathrm{b}}}=\gamma_{0} \sum_{\forall k, m} \Re\left\{\alpha_{f} k\left(\mathbf{a}_{\mathrm{g}, k}^{\mathrm{H}} \breve{\mathbf{F}}_{k, m} \dot{\mathbf{a}}_{\mathrm{g}, \theta, k}^{\mathrm{H}}\right)\left(\mathbf{a}_{\mathrm{u}, k}^{\mathrm{H}} \breve{\mathbf{W}}_{k, m} \mathbf{a}_{\mathrm{u}, k}\right)\right\}, \\
& J_{\theta_{\mathrm{g}} f_{\mathrm{g}}}=\gamma_{0} \sum_{\forall k, m} \Re\left\{\left(\mathbf{a}_{\mathrm{g}, k}^{\mathrm{H}} \dot{\mathbf{G}}_{k, m} \dot{\mathbf{a}}_{\mathrm{g}, \theta, k}\right)\left(\mathbf{a}_{\mathrm{u}, k}^{\mathrm{H}} \breve{\mathbf{W}}_{k, m} \mathbf{a}_{\mathrm{u}, k}\right)\right\}, \\
& J_{\phi_{\mathrm{g}}}=\gamma_{0} \sum_{\forall k, m} \Re\left\{\left(\dot{\mathbf{a}}_{\mathrm{g}, \phi, k}^{\mathrm{H}} \breve{\mathbf{F}}_{k, m} \dot{\mathbf{a}}_{\mathrm{g}, \phi, k}\right)\left(\mathbf{a}_{\mathrm{u}, k}^{\mathrm{H}} \breve{\mathbf{W}}_{k, m} \mathbf{a}_{\mathrm{u}, k}\right)\right\}, \\
& J_{\phi_{\mathrm{g}} \theta_{\mathrm{u}}}=\gamma_{0} \sum_{\forall k, m} \Re\left\{\left(\mathbf{a}_{\mathrm{g}, k}^{\mathrm{H}} \breve{\mathbf{F}}_{k, m} \dot{\mathbf{a}}_{\mathrm{g}, \phi, k}\right)\left(\mathbf{a}_{\mathrm{u}, k}^{\mathrm{H}} \breve{\mathbf{W}}_{k, m} \dot{\mathbf{a}}_{\mathrm{u}, \theta, k}\right)\right\}, \\
& J_{\phi_{\mathrm{g}} \phi_{\mathrm{u}}}=\gamma_{0} \sum_{\forall k, m} \Re\left\{\left(\mathbf{a}_{\mathrm{g}, k}^{\mathrm{H}} \breve{\mathbf{F}}_{k, m} \dot{\mathbf{a}}_{\mathrm{g}, \phi, k}\right)\left(\mathbf{a}_{\mathrm{u}, k}^{\mathrm{H}} \breve{\mathbf{W}}_{k, m} \dot{\mathbf{a}}_{\mathrm{u}, \phi, k}\right)\right\}, \\
& J_{\phi_{\mathrm{g}} \tau_{\mathrm{b}}}=\gamma_{0} \sum_{\forall k, m} \Re\left\{\alpha_{f} k\left(\mathbf{a}_{\mathrm{g}, k}^{\mathrm{H}} \breve{\mathbf{F}}_{k, m} \dot{\mathbf{a}}_{\mathrm{g}, \phi, k}\right)\left(\mathbf{a}_{\mathrm{u}, k}^{\mathrm{H}} \breve{\mathbf{W}}_{k, m} \mathbf{a}_{\mathrm{u}, k}\right)\right\}, \\
& J_{\phi_{\mathrm{g}} f_{\mathrm{g}}}=\gamma_{0} \sum_{\forall k, m} \Re\left\{\left(\mathbf{a}_{\mathrm{g}, k}^{\mathrm{H}} \dot{\mathbf{G}}_{k, m} \dot{\mathbf{a}}_{\mathrm{g}, \phi, k}\right)\left(\mathbf{a}_{\mathrm{u}, k}^{\mathrm{H}} \breve{\mathbf{W}}_{k, m} \mathbf{a}_{\mathrm{u}, k}\right)\right\}, \\
& J_{\theta_{\mathrm{u}}}=\gamma_{0} \sum_{\forall k, m} \Re\left\{\left(\mathbf{a}_{\mathrm{g}, k}^{\mathrm{H}} \breve{\mathbf{F}}_{k, m} \mathbf{a}_{\mathrm{g}, k}\right)\left(\dot{\mathbf{a}}_{\mathrm{u}, \theta, k}^{\mathrm{H}} \breve{\mathbf{W}}_{k, m} \dot{\mathbf{a}}_{\mathrm{u}, \theta, k}\right)\right\},
\end{aligned}
$$




$$
\begin{aligned}
J_{\theta_{\mathrm{u}} \phi_{\mathrm{u}}}= & \gamma_{0} \sum_{\forall k, m} \Re\left\{\left(\mathbf{a}_{\mathrm{g}, k}^{\mathrm{H}} \breve{\mathbf{F}}_{k, m} \mathbf{a}_{\mathrm{g}, k}\right)\left(\dot{\mathbf{a}}_{\mathrm{u}, \theta, k}^{\mathrm{H}} \breve{\mathbf{W}}_{k, m} \dot{\mathbf{a}}_{\mathrm{u}, \phi, k}\right)\right\}, \\
J_{\theta_{\mathrm{u}} \tau_{\mathrm{bg}}}= & \gamma_{0} \sum_{\forall k, m} \Re\left\{\alpha_{f} k\left(\mathbf{a}_{\mathrm{g}, k}^{\mathrm{H}} \breve{\mathbf{F}}_{k, m} \mathbf{a}_{\mathrm{g}, k}\right)\left(\dot{\mathbf{a}}_{\mathrm{u}, \theta, k}^{\mathrm{H}} \breve{\mathbf{W}}_{k, m} \mathbf{a}_{\mathrm{u}, k}\right)\right\}, \\
J_{\theta_{\mathrm{u}} f_{\mathrm{dg}}}= & \gamma_{0} \sum_{\forall k, m} \Re\left\{\left(\mathbf{a}_{\mathrm{g}, k}^{\mathrm{H}} \dot{\mathbf{G}}_{k, m} \mathbf{a}_{\mathrm{g}, k}\right)\left(\dot{\mathbf{a}}_{\mathrm{u}, \theta, k}^{\mathrm{H}} \breve{\mathbf{W}}_{k, m} \mathbf{a}_{\mathrm{u}, k}\right)\right\}, \\
J_{\phi_{\mathrm{u}}}= & \gamma_{0} \sum_{\forall k, m} \Re\left\{\left(\mathbf{a}_{\mathrm{g}, k}^{\mathrm{H}} \breve{\mathbf{F}}_{k, m} \mathbf{a}_{\mathrm{g}, k}\right)\left(\dot{\mathbf{a}}_{\mathrm{u}, \phi, k}^{\mathrm{H}} \breve{\mathbf{W}}_{k, m} \dot{\mathbf{a}}_{\mathrm{u}, \phi, k}\right)\right\}, \\
J_{\phi_{\mathrm{u}} \tau_{\mathrm{bg}}}= & \gamma_{0} \sum_{\forall k, m} \Re\left\{\alpha_{f} k\left(\mathbf{a}_{\mathrm{g}, k}^{\mathrm{H}} \breve{\mathbf{F}}_{k, m} \mathbf{a}_{\mathrm{g}, k}\right)\left(\dot{\mathbf{a}}_{\mathrm{u}, \phi, k}^{\mathrm{H}} \breve{\mathbf{W}}_{k, m} \mathbf{a}_{\mathrm{u}, k}\right)\right\}, \\
J_{\phi_{\mathrm{u}} f_{\mathrm{dg}}}= & \gamma_{0} \sum_{\forall k, m} \Re\left\{\left(\mathbf{a}_{\mathrm{g}, k}^{\mathrm{H}} \dot{\mathbf{G}}_{k, m} \mathbf{a}_{\mathrm{g}, k}\right)\left(\dot{\mathbf{a}}_{\mathrm{u}, \phi, k}^{\mathrm{H}} \breve{\mathbf{W}}_{k, m} \mathbf{a}_{\mathrm{u}, k}\right)\right\}, \\
J_{\tau_{\mathrm{bg}}}= & \gamma_{0}\left|\alpha_{f}\right|^{2} \sum_{\forall k, m} \Re\left\{k^{2}\left(\mathbf{a}_{\mathrm{g}, k}^{\mathrm{H}} \breve{\mathbf{F}}_{k, m} \mathbf{a}_{\mathrm{g}, k}\right)\left(\mathbf{a}_{\mathrm{u}, k}^{\mathrm{H}} \breve{\mathbf{W}}_{k, m} \mathbf{a}_{\mathrm{u}, k}\right)\right\}, \\
J_{\tau_{\mathrm{b} g} f_{\mathrm{dg}}}= & -\gamma_{0} \sum_{\forall k, m} \Re\left\{\alpha_{f} k\left(\mathbf{a}_{\mathrm{g}, k}^{\mathrm{H}} \mathbf{a}_{\mathrm{g}, k}\right)\left(\mathbf{a}_{\mathrm{u}, k}^{\mathrm{H}} \breve{\mathbf{W}}_{k, m} \mathbf{a}_{\mathrm{u}, k}\right)\right\}, \\
J_{f_{\mathrm{dg}}}= & \gamma_{0} \sum_{\forall k, m} \Re\left\{\left(\mathbf{a}_{\mathrm{g}, k}^{\mathrm{H}} \ddot{\mathbf{G}}_{k, m} \mathbf{a}_{\mathrm{g}, k}\right)\left(\mathbf{a}_{\mathrm{u}, k}^{\mathrm{H}} \breve{\mathbf{W}}_{k, m} \mathbf{a}_{\mathrm{u}, k}\right)\right\} .
\end{aligned}
$$

\section{APPENDIX B}

FIM of SATELlite PARAMETERS

Starting with $\mu_{s}(t)=\sqrt{P_{\mathrm{s}}} x_{s}\left(t-\tau_{\mathrm{b}_{s}}\right) \mathrm{e}^{j 2 \pi f_{d_{s}} t}$ and noting that $\frac{\partial}{\partial \tau_{\mathrm{b} s}} x_{s}\left(t-\tau_{\mathrm{b}_{s}}\right)=-\dot{x}_{s}\left(t-\tau_{\mathrm{b}_{s}}\right)$, then

$$
\begin{aligned}
& \frac{\partial \mu_{s}(t)}{\partial \tau_{\mathrm{b} s}}=-\sqrt{P_{\mathrm{s}}} \dot{x}_{s}\left(t-\tau_{\mathrm{b}_{s}}\right) \mathrm{e}^{j 2 \pi f_{d_{s}} t}, \\
& \frac{\partial \mu_{s}(t)}{\partial f_{\mathrm{d}_{\mathrm{b}}}}=j 2 \pi t \sqrt{P_{\mathrm{s}}} x_{s}\left(t-\tau_{\mathrm{b}_{s}}\right) \mathrm{e}^{j 2 \pi f_{d_{s}} t} .
\end{aligned}
$$

Consequently, from (11),

$$
\begin{aligned}
J_{\tau_{\mathrm{b} s}} & =\frac{P_{\mathrm{s}}}{N_{0}} \int_{0}^{T_{\mathrm{so}}}\left|\dot{x}_{s}\left(t-\tau_{\mathrm{b}_{s}}\right)\right|^{2} \mathrm{~d} t \\
& =\frac{P_{\mathrm{s}}}{N_{0}} \int_{-W / 2}^{W / 2}(2 \pi f)^{2}|X(f)|^{2} \mathrm{~d} f, \\
& =\frac{N_{\mathrm{so}} P_{\mathrm{s}}}{N_{0}} \int_{-W / 2}^{W / 2}(2 \pi f)^{2}|R(f)|^{2} \mathrm{~d} f, \\
& =\frac{4 \pi^{2} P_{\mathrm{s}} T_{\mathrm{so}}}{N_{0}} W_{\mathrm{eff}}^{2},
\end{aligned}
$$

where

$$
W_{\text {eff }}^{2} \triangleq \frac{1}{T_{\mathrm{c}}} \int_{-W / 2}^{W / 2} f^{2}|R(f)|^{2} \mathrm{~d} f .
$$

(18b) follows from Parseval's theorem, while (18c) follows from (7). For a rectangular pulse shape, it can be shown that $W_{\text {eff }}^{2}=W^{2} /\left(2 \pi^{2}\right)$. Similarly,

$$
J_{f_{\mathrm{d} s}}=\frac{4 \pi^{2} P_{\mathrm{s}}}{N_{0}} \int_{0}^{T_{\mathrm{so}}} t^{2}\left|x_{\mathrm{s}}\left(t-\tau_{\mathrm{b}_{s}}\right)\right|^{2} \mathrm{~d} t
$$

By the expansion of the summation in (7), and a change of variables, it can be shown that

$$
J_{f_{\mathrm{ds}}}=\frac{4 \pi^{2} P_{\mathrm{s}} T_{\mathrm{so}} T_{\mathrm{eff}}^{2}}{N_{0}}
$$

where

$$
T_{\text {eff }}^{2} \triangleq \int_{0}^{T_{\mathrm{c}}}\left\{\frac{1}{N_{\mathrm{so}}} \sum_{\ell=0}^{N_{\mathrm{so}}-1}\left(t+\ell T_{\mathrm{c}}\right)^{2}\right\}|r(t)|^{2} \mathrm{~d} t
$$

For a rectangular pulse, $T_{\text {eff }}^{2}=T_{\mathrm{so}}^{2} / 12$.

Note that $J_{\tau_{\mathrm{b} s} f_{\mathrm{d} s}}=0$, because the integrand below is imaginary

$$
J_{\tau_{\mathrm{bs}} f_{\mathrm{d}_{s}}}=\frac{-2 \pi P_{\mathrm{s}}}{N_{0}} \int_{0}^{T_{\mathrm{so}}} \Re\left\{j t\left|x_{s}\left(t-\tau_{\mathrm{b}_{s}}\right)\right|^{2}\right\} \mathrm{d} t=0 .
$$

\section{APPENDIX C}

\section{NON-ZERo ENTRIES OF TRANSFORMation Matrices}

\section{A. $5 G$ Parameter Transformation Matrix $\mathbf{T}_{\mathrm{g}}$}

Defining $\overline{\mathbf{p}}_{g} \triangleq \mathbf{p}-\mathbf{p}_{g}=\left[\bar{p}_{g, x}, \bar{p}_{g, y}, \bar{p}_{g, z}\right]^{\mathrm{T}}$ and $\overline{\mathbf{v}}_{g} \triangleq$ $\mathbf{v}-\mathbf{v}_{g}=\left[\bar{v}_{g, x}, \bar{v}_{g, y}, \bar{v}_{g, z}\right]^{\mathrm{T}}$, then the non-zero elements in the transformation matrix $\mathbf{T}_{\mathrm{g}}$ can be shown to be

$$
\begin{aligned}
& \frac{\partial \theta_{\mathrm{g}}}{\partial \mathbf{p}}=\frac{\left[\begin{array}{lll}
\bar{p}_{x} \bar{p}_{z} & \bar{p}_{y} \bar{p}_{z}-\left(\bar{p}_{x}+\bar{p}_{y}\right)^{2}
\end{array}\right]^{\mathrm{T}}}{\left\|\overline{\mathbf{p}}_{g}\right\|^{2} \sqrt{\bar{p}_{x}^{2}+\bar{p}_{y}^{2}}}, \\
& \frac{\partial \phi_{\mathrm{g}}}{\partial \mathbf{p}}=\frac{1}{\bar{p}_{x}^{2}+\bar{p}_{y}^{2}}\left[\begin{array}{lll}
-\bar{p}_{y} & \bar{p}_{x} & 0
\end{array}\right]^{\mathrm{T}}, \\
& \frac{\partial \theta_{\mathrm{u}}}{\partial \mathbf{p}}=-\frac{\partial \theta_{\mathrm{g}}}{\partial \overline{\mathbf{p}}_{g}} \\
& \frac{\partial \phi_{\mathrm{u}}}{\partial \mathbf{p}}=\frac{\partial \phi_{\mathrm{g}}}{\partial \overline{\mathbf{p}}_{g}}, \\
& \frac{\partial f_{\mathrm{d}_{\mathrm{g}}}}{\partial \mathbf{p}}=\frac{\left(\overline{\mathbf{v}}_{g}^{\mathrm{T}} \overline{\mathbf{p}}_{g}\right) \overline{\mathbf{p}}_{g}-\left\|\overline{\mathbf{p}}_{g}\right\|^{2} \overline{\mathbf{v}}_{g}}{\lambda_{g}\left\|\overline{\mathbf{p}}_{g}\right\|^{3}}, \\
& \frac{\partial \tau_{\mathrm{b}_{\mathrm{g}}}}{\partial \mathbf{p}}=\frac{\overline{\mathbf{p}}_{g}}{c\|\mathbf{p}\|}+\frac{b_{\mathrm{u}}}{f_{g}} \frac{\partial f_{\mathrm{d}_{\mathrm{g}}}}{\partial \mathbf{p}} \\
& \frac{\partial f_{\mathrm{d}_{\mathrm{g}}}}{\partial \mathbf{v}}=-\frac{\overline{\mathbf{p}}_{g}}{\lambda_{g}\|\mathbf{p}\|}, \\
& \frac{\partial \tau_{\mathrm{b}_{\mathrm{g}}}}{\partial \mathbf{v}}=\frac{b_{\mathrm{u}}}{f_{g}} \frac{\partial f_{\mathrm{dg}_{\mathrm{g}}}}{\partial \mathbf{v}} \\
& \frac{\partial \tau_{\mathrm{b}_{\mathrm{g}}}}{\partial b_{\mathrm{u}}}=1+\frac{f_{\mathrm{d}_{\mathrm{g}}}}{f_{g}} .
\end{aligned}
$$

\section{B. $5 G$ Parameter Transformation Matrix $\mathbf{T}_{\mathrm{g}}$}

Defining $\overline{\mathbf{p}}_{s} \triangleq \mathbf{p}-\mathbf{p}_{\mathrm{s}}$ and $\overline{\mathbf{v}}_{s} \triangleq \mathbf{v}-\mathbf{v}_{\mathrm{s}}$, then the non-zero elements in the transformation matrix $\mathbf{T}_{s}$ can be shown to be

$$
\begin{aligned}
\frac{\partial f_{\mathrm{d}_{s}}}{\partial \mathbf{p}} & =\frac{\left(\overline{\mathbf{v}}_{s}^{\mathrm{T}} \overline{\mathbf{p}}_{s}\right) \overline{\mathbf{p}}_{s}-\left\|\overline{\mathbf{p}}_{s}\right\|^{2} \overline{\mathbf{v}}_{s}}{\lambda_{s}\left\|\overline{\mathbf{p}}_{s}\right\|^{3}}, \\
\frac{\partial \tau_{\mathrm{b}_{\mathrm{s}}}}{\partial \mathbf{p}} & =\frac{\overline{\mathbf{p}}_{s}}{c\left\|\overline{\mathbf{p}}_{s}\right\|}+\frac{b_{\mathrm{u}}}{f_{s}} \frac{\partial f_{\mathrm{d}_{s}}}{\partial \mathbf{p}} \\
\frac{\partial f_{\mathrm{d}_{s}}}{\partial \mathbf{v}} & =-\frac{\overline{\mathbf{p}}_{s}}{\lambda_{s}\left\|\overline{\mathbf{p}}_{s}\right\|}, \\
\frac{\partial \tau_{\mathrm{b}_{s}}}{\partial \mathbf{v}} & =\frac{b_{\mathrm{u}}}{f_{\mathrm{s}}} \frac{\partial f_{\mathrm{d}_{s}}}{\partial \mathbf{v}} \\
\frac{\partial \tau_{\mathrm{b}_{\mathrm{s}}}}{\partial b_{\mathrm{u}}} & =1+\frac{f_{\mathrm{d}_{s}}}{f_{s}} .
\end{aligned}
$$




\section{REFERENCES}

[1] S. Bisnath, M. U. de Haag, D. W. Diggle, D. Milbert, and T. Walter, "Differential GNSS and Precise Point Positioning," in Understanding GPS/GNSS: Principles and Applications, E. D. Kaplan and C. J. Hegarty, Eds. Boston: Artech House, 2017, ch. 12, pp. 709-788.

[2] J. B. Bullock and M. King, "Integration of GNSS with Other Sensors and Network Assistance," in Understanding GPS/GNSS: Principles and Applications, E. D. Kaplan and C. J. Hegarty, Eds. Boston: Artech House, 2017, ch. 13, pp. 789-914.

[3] J. G. Andrews, S. Buzzi, W. Choi, S. V. Hanly, A. Lozano, A. Soong, and J. C. Zhang, "What will 5G be?" IEEE J. Sel. Areas Commun., vol. 32, no. 6, pp. 1065-1082, June 2014.

[4] T. S. Rappaport, S. Sun, R. Mayzus, H. Zhao, Y. Azar, K. Wang, G. N. Wong, J. K. Schulz, M. Samimi, and F. Gutierrez, "Millimeter wave mobile commun. for $5 \mathrm{G}$ cellular: It will work!" IEEE Access, vol. 1 , pp. 335-349, 2013.

[5] I. F. Akyildiz, S. Nie, S.-C. Lin, and M. Chandrasekaran, "5G roadmap: 10 key enabling technologies," Computer Networks, vol. 106, pp. 17 48, 2016.

[6] R. W. Heath, N. González-Prelcic, S. Rangan, W. Roh, and A. M. Sayeed, "An overview of signal processing techniques for millimeter wave MIMO systems," IEEE J. Sel. Topics in Signal Process., vol. 10, no. 3, pp. 436-453, April 2016

[7] R. D. Taranto, S. Muppirisetty, R. Raulefs, D. Slock, T. Svensson, and H. Wymeersch, "Location-aware communications for 5G networks: How location information can improve scalability, latency, and robustness of 5G," IEEE Signal Process. Mag, vol. 31, no. 6, pp. 102-112, Nov 2014.
[8] A. Guerra, F. Guidi, and D. Dardari, "Single-anchor localization and orientation performance limits using massive arrays: MIMO vs. beamforming," IEEE Transactions on Wireless Communications, vol. 17, no. 8, pp. 5241-5255, Aug 2018.

[9] Z. Abu-Shaban, X. Zhou, T. Abhayapala, G. Seco-Granados, and H. Wymeersch, "Error bounds for uplink and downlink 3D localization in 5G millimeter wave systems," IEEE Transactions on Wireless Communications, vol. 17, no. 8, pp. 4939-4954, Aug 2018.

[10] R. Mendrzik, H. Wymeersch, G. Bauch, and Z. Abu-Shaban, "Harnessing NLOS components for position and orientation estimation in 5G millimeter wave MIMO," IEEE Transactions on Wireless Communications, vol. 18, no. 1, pp. 93-107, Jan 2019.

[11] S. M. Kay, Fundamentals of Statistical Signal Processing: Estimation Theory. NJ, USA: Prentice-Hall, Inc., 1993.

[12] S. Barbarossa, Multiantenna wireless communications systems. Artech House Publishers, 2005.

[13] European Sapce Agency. GPS Space Segment Navipedia. Accessed: Dec 13, 2019. [Online]. Available: https://gssc.esa.int/navipedia/index.php

[14] M. Khatun, H. Mehrpouyan, D. Matolak, and I. Guvenc, "Millimeter wave systems for airports and short-range aviation communications: A survey of the current channel models at mmwave frequencies," in 2017 IEEE/AIAA 36th Digital Avionics Systems Conference (DASC), Sep. 2017, pp. 1-8. 